the game and summarised data showing progress was favoured. Parents were incentivised by this opportunity for their child to receive help and take responsibility for therapy. Appearance/comfort: The devices were programmed to display the time, in response to requests from YP. Wearing two devices was mildly perturbing for older participants as this would be considered unusual, though all were prepared to do it for the project duration. Access to participants: Recruitment to the workshops through advertising and patient groups was challenging; however, in the planned study, we will recruit through the NHS.

Conclusion The participatory design process informed plans for the proof of concept stage of the study, hopefully leading to a product and approach that will be fun, easy to integrate into everyday life, and have the capacity to increase use of the affected arm and hand.

\section{G49(P) RCPCH STROKE IN CHILDHOOD GUIDELINES: ARE THESE BEING FOLLOWED?}

YC Collins-Sawaragi, H Walker, J Sanperaiglesias, S Byrne, E Wraige, D Lumsden, S Tang. Paediatric Neurology, Evelina London Children's Hospital, London, UK

\subsection{6/archdischild-2020-rcpch.35}

Aims We investigate if the RCPCH Stroke in Childhood Guidelines (2017) are being followed in paediatric patients presenting with a 'brain attack'.

Methods We retrospectively collected two cohorts of paediatric patients aged over 2 years old presenting with symptoms suggestive of stroke ('brain attack') over 6 months between March and August 2019. Cohort 1 were patients from a Tertiary Hospital A\&E department that were identified through the Symphony electronic database. Cohort 2 were from patient referrals to the Tertiary Paediatric Neurology Department documented in the electronic database. Patient demographics, symptoms, NIHSS (National Institute of Health Stroke Scale), investigations, time to brain scan, thrombolysis and thrombectomy rates and final diagnosis were collected.

Results 33 patients in Cohort 1 and 32 patients in Cohort 2 had symptoms that met possible stroke diagnosis. Mean age of all patients were 8.9 years (36 male, 29 female). The top 3 presenting symptoms were focal seizures (20/65), speech disturbance $(14 / 65)$ and headache (13/65). Only 6 patients had NIHSS calculated. Cranial imaging was performed within 1 hour of admission in only $1 / 33$ in the A\&E cohort and 2/32 in the external referral cohort. $9 / 65$ patients had a diagnosis of stroke: 2 transient ischaemic attacks, 5 ischaemic strokes, 1 venous stroke and 1 haemorrhagic stroke. 28/65 patients had other diagnoses requiring urgent treatment such as seizure disorders and infection. In total 2 patients were evaluated for thrombolysis - 1 patient received thrombolysis under the care of the adult stroke team. 2 patients were referred for thrombectomy but none were suitable candidates (1 symptom resolution before thrombectomy, 1 high risk of complications due to anticoagulation post-operatively).

Conclusion RCPCH Stroke in Childhood Guidelines are not consistently being followed, particularly with regards to calculation of NIHSS and delays in obtaining brain imaging. If guidelines are not followed, paediatric stroke patients and windows of treatment with thrombolysis/thrombectomy may be missed. This highlights the importance of having a paediatric stroke pathway in place.

\section{G50(P) GETTING TO THE HEART OF THE WHITE MATTER}

${ }^{1}$ I Morgan, ${ }^{1} \mathrm{C}$ Herbert, ${ }^{2} \mathrm{~K}$ Forbes, ${ }^{3} \mathrm{M}$ O'Regan, ${ }^{4} \mathrm{~L}$ Hunter, ${ }^{1} \mathrm{~K}$ Forrest. ${ }^{1}$ Paediatric Neurology, RHC, Glasgow, UK; ${ }^{2}$ Paediatric Radiology, RHC, Glasgow, UK; ${ }^{3}$ Paediatric Neurology, Our Lady's Children's Hospital, Dublin, Ireland; ${ }^{4}$ Paediatric Cardiology, RHC, Glasgow, UK

\subsection{6/archdischild-2020-rcpch.36}

Objective Acute Disseminated Encephalomyelitis (ADEM) is an immune mediated inflammatory CNS disorder, predominantly affecting white matter, with a wide differential. ${ }^{1}$ Here we describe a rare mimic of ADEM that is essential to consider in order to avoid catastrophic outcome.

Case History A 12-year old girl presented with a 2 day history of confusion, dysarthria, ataxia and left-sided squint, preceded by 12 days of being generally unwell, with headache and lethargy. Examination confirmed encephalopathy and left third cranial nerve palsy.

MRI brain was suggestive of ADEM and MRI spine was normal. A recommended work up for $\mathrm{ADEM}^{1}$ was performed. Rapid resolution of her symptoms occurred following treatment with intravenous methylprednisolone for three days.

2 years later she presented with acute left lower limb ischaemia and underwent emergency embolectomy of a popliteal arterial obstruction; with myxomatous material identified. Preoperative echocardiogram confirmed left atrial mass which was then surgically removed. Pathology confirmed an atrial myxoma (AM).

Retrospective review of her initial MRI images concluded that embolic phenomena from the AM was the most likely explanation of her first presentation.

Conclusions AM is a very rare primary cardiac tumour and left sided AM can embolise to the cerebrovascular system. $12 \%$ of adults with AM present with neurological symptoms, and this can mimic multiple sclerosis. AM presenting with acute neurological symptoms masquerading as ADEM in paediatrics has not previously been reported. Early identification of $\mathrm{AM}$ is important as, untreated, it can cause multiple embolic events and sudden cardiac death. Careful follow up is essential as late neurological complications (including cerebral arterial aneurysms) are recognised.

This case highlights that ADEM is a diagnosis of exclusion, and that mimics for acute focal neurology with encephalopathy and T2 hyperintensities on MRI require careful consideration, including embolic phenomena. Clinical examination alone does not exclude AM and consideration of echocardiography is recommended.

\section{REFERENCE}

1. Differential diagnosis and evaluation in paediatric inflammatory demyelinating disorders. Neurology 2016 (Supp|2);87:S28-37.

\section{G51(P) INTERACTION OF CANNABIDIOL (CBD) WITH OTHER ANTISEIZURE MEDICATIONS (ASMS)}

${ }^{1}$ CGS Gilmartin, ${ }^{2} Z$ Dowd, 2 PD Harijan. 'School of Clinical Medicine, University of Cambridge, Cambridge, UK; ${ }^{2}$ Department of Paediatric Neurology, Cambridge University Hospitals NHS Trust, Cambridge, UK

\subsection{6/archdischild-2020-rcpch.37}

Objective Cannabidiol is efficacious as an adjunctive treatment in children with epilepsy associated with Dravet and LennoxGastaut syndromes. As cannabidiol is currently co-prescribed with other antiseizure medications (ASMs), a literature review 